Abstract 198 Table 2 Diagnostic performance regarding detection of myometrial and cervial invasion

\begin{tabular}{|c|c|c|c|c|c|c|c|c|c|}
\hline & $\mathbf{T P}$ & FP & $\mathbf{F N}$ & $\mathbf{T N}$ & $\begin{array}{c}\text { Sensitivity } \\
\%\end{array}$ & $\begin{array}{c}\text { Specificity } \\
\% \\
\end{array}$ & $\begin{array}{c}\text { Accuracy } \\
\% \\
\end{array}$ & $\mathbf{P P V}^{*}$ & $\mathbf{N P V}^{* *}$ \\
\hline \multicolumn{10}{|l|}{ FICO } \\
\hline I & 34 & 2 & 18 & 14 & $65.4 \%$ & $78.5 \%$ & $70.5 \%$ & $94.4 \%$ & \\
\hline II & 5 & 3 & 4 & 56 & $55.5 \%$ & $94.9 \%$ & $89.7 \%$ & $62.5 \%$ & \\
\hline III & 3 & 3 & 4 & 58 & $42.8 \%$ & $95.1 \%$ & $89.7 \%$ & $50 \%$ & $93.5 \%$ \\
\hline \multicolumn{10}{|c|}{ Myometrial Invasion } \\
\hline No & 1 & 18 & 0 & 50 & $100 \%$ & $73.5 \%$ & $75 \%$ & $5.2 \%$ & $100 \%$ \\
\hline$<50 \%$ & 19 & 8 & 19 & 22 & $50 \%$ & $73.3 \%$ & $60 \%$ & $70.3 \%$ & $53.6 \%$ \\
\hline$\geq 50 \%$ & 20 & 2 & 9 & 37 & $68.9 \%$ & $94.8 \%$ & $83.8 \%$ & $90.9 \%$ & $80.4 \%$ \\
\hline \multicolumn{10}{|c|}{ Node involvement } \\
\hline No & 59 & 3 & 3 & 3 & $95.1 \%$ & $50 \%$ & $91.1 \%$ & $95.1 \%$ & $50 \%$ \\
\hline Yes & 3 & 3 & 3 & 59 & $50 \%$ & $95.1 \%$ & $91.1 \%$ & $50 \%$ & $95.1 \%$ \\
\hline \multicolumn{10}{|c|}{ Cervical stromal involvement } \\
\hline No & 53 & 5 & 1 & 9 & $98.1 \%$ & $64.3 \%$ & $91.1 \%$ & $91.4 \%$ & $90 \%$ \\
\hline Yes & 9 & 1 & 5 & 53 & $64.3 \%$ & $98.1 \%$ & $91.1 \%$ & $90 \%$ & $91.4 \%$ \\
\hline
\end{tabular}

"Positive predictive value; ${ }^{* *}$ Negative predictive value

pathologist.The results were compared with the final histopathology report of surgical staging.

Result(s)*

Results Included were a total of 68 women with endometrioid adenocarcinoma of the uterus, most $(76 \%)$ with stage I disease. Levels of serum HE4 greater than 140PM and CA125 greater than $35 \mathrm{ku} / \mathrm{L}$ were observed in $12(17 \%)$ and 26 $(38.2 \%)$ of patients respectively whose greater proportion were cases with deep myometrial invasion and high grade tumor. In the evaluation of deep tumoral invasion (> 50\%) of the myometrium sensitivity, specificity, and diagnostic accuracy of MRI were $68.9 \%, 94.8 \%$ and $83.8 \%$ respectively. For lymph node involvement these values were 50\%, 95.1\% and $91.1 \%$ respectively and for cervical stromal involvement were $64.3 \%, 98.1 \%$ and $91.1 \%$ respectively.

Conclusion* Higher stage, deep myometrial invasion, and lymph node or cervical stromal involvement increase diagnostic accuracy of MRI. Higher levels of HE4 and CA125 were observed in patients with deep myometrial invasion and higher grade of tumor.

\section{ENDOMETRIAL CARCINOMA MOLECULAR SUBTYPE CORRELATES WITH THE PRESENCE OF LYMPH NODE METASTASES}

${ }^{1}$ EF Thompson*, ${ }^{2} \mathrm{~A}$ Jamieson, ${ }^{3} \mathrm{~J}$ Huvila, ${ }^{4} \mathrm{~S}$ Leung, ${ }^{1} \mathrm{~A}$ Lum, ${ }^{5} \mathrm{C}$ Morin, ${ }^{5} \mathrm{~K}$ Ennour-Idrissi, ${ }^{6} \mathrm{~A}$ Sebastianelli, ${ }^{6} \mathrm{MC}$ Renaud, ${ }^{6} \mathrm{~J}$ Gregoire, ${ }^{6} \mathrm{M}$ Plante, ${ }^{1} \mathrm{D}$ Huntsman, ${ }^{7} \mathrm{CB}$ Gilks, ${ }^{5} \mathrm{~K}$ Grondin, 2J Mcalpine. 'The University of British Columbia, Molecular Oncology, Vancouver, Canada; ${ }^{2}$ The University of British Columbia, Gynaecologic Oncology, Vancouver, Canada; ${ }^{3}$ University of Turku, Institute of Biomedicine, Turku, Finland; ${ }^{4}$ The University of British Columbia, Genetic Pathology Evaluation Centre, Vancouver, Canada; ${ }^{5}$ Chu De Québec-Université Laval, Pathology, Québec, Canada; ${ }^{6}$ Chu De Québec-Université Laval, Gynecology Oncology Service, Québec, Canada; ${ }^{7} T h e$ University of British Columbia, Vancouver General Hospital, Pathology and Laboratory Medicine, Vancouver, Canada
Introduction/Background* The role of lymph node (LN) assessment in endometrial cancer (EC) has been a subject of debate for decades, with significant variation in use between centres. Molecular classification of EC provides objective, prognostic information and be performed on diagnostic endometrial biopsy specimens. The Proactive Molecular Risk Classifier for Endometrial Cancer (ProMisE) uses targeted nextgeneration sequencing to detect pathogenic POLE exonuclease domain mutations (POLEmut) and immunohistochemistry to evaluate for the presence of mismatch repair deficiency (MMRd), TP53 mutations (p53abn) or tumours with no specific molecular profile (NSMP/p53wt). Herein, we assessed the association between EC molecular subtype and LN metastases in a single institutional cohort with a uniform approach to LN assessment.

Methodology All ECs treated with primary surgery from a single institution in 2015 underwent ProMisE molecular subtyping and collection of clinicopathologic and outcomes data.

Result(s)* Complete pelvic and para-aortic lymphadenectomies were performed in 171 of 172 consecutive cases of EC. The distribution of ProMisE subtypes and clinicopathologic features associated with molecular subtype are outlined in table 1. The p53abn subtype was observed across a range of EC histotypes, including low grade endometrioid endometrial carcinoma. LN metastases were found in $31 / 171$ (18.1\%) patients: pelvic only in $83.9 \%$ and pelvic plus para-aortic in 16.1\%. LN metastases included macrometastases (19/31), micrometastases (5/31), and isolated tumour cells (ITCs) (7/ 31).

Molecular subtype was significantly associated with LN metastases $(p=0.004)$; there was a strong association between LN metastases and p53abn EC (nodal involvement in $44.8 \%$ of cases). LN metastases were observed in $14.2 \%$ of POLEmut, $14.9 \%$ of MMRd, and $10.8 \%$ of NSMP EC. 
Abstract 223 Table 1 Summary of the clinicopathologic features by ProMisE molecular subtype

\begin{tabular}{|c|c|c|c|c|c|}
\hline Variable & Total & POLEmut & MMRd & NSMP & p53abn \\
\hline Total & 172 & 21 (12.2\%) & $47(27.3 \%)$ & $74(43.0 \%)$ & 30 (17.4\%) \\
\hline $\begin{array}{l}\text { Age at diagnosis } \\
<60 \text { years } \\
\geq 60 \text { years }\end{array}$ & $\begin{array}{c}70(40.7 \%) \\
102(59.3 \%)\end{array}$ & $\begin{array}{c}12(57.1 \%) \\
9(42.9 \%)\end{array}$ & $\begin{array}{l}18(38.3 \%) \\
29(61.7 \%)\end{array}$ & $\begin{array}{l}38(51.4 \%) \\
36(48.6 \%)\end{array}$ & $\begin{array}{c}2(6.6 \%) \\
28(93.3 \%)\end{array}$ \\
\hline $\begin{array}{l}\text { Histotype } \\
\text { Endometrioid } \\
\text { Non-endometrioid } \\
\text {-Serous } \\
\text {-Clear cell } \\
\text {-Carcinosarcoma } \\
\text {-Mixed }\end{array}$ & $\begin{array}{c}137(79.7 \%) \\
35(20.3 \%) \\
15(8.7 \%) \\
2(1.2 \%) \\
8(4.7 \%) \\
10(5.8 \%)\end{array}$ & $\begin{array}{l}18(85.7 \%) \\
3(14.3 \%) \\
0 \\
0 \\
0 \\
3(14.3 \%)\end{array}$ & $\begin{array}{l}45(95.7 \%) \\
2(4.3 \%) \\
1(2.1 \%) \\
0 \\
0 \\
1(2.1 \%)\end{array}$ & $\begin{array}{l}71(95.9 \%) \\
3(4.1 \%) \\
0 \\
1(1.4 \%) \\
0 \\
2(2.7 \%)\end{array}$ & $\begin{array}{c}3(10.0 \%) \\
27(90.0 \%) \\
14(46.7 \%) \\
1(3.3 \%) \\
8(26.7 \%) \\
4(13.3 \%)\end{array}$ \\
\hline $\begin{array}{l}\text { Tumour grade } \\
\text { low grade } \\
\text { high grade }\end{array}$ & $\begin{array}{c}129(75 \%) \\
43(25 \%)\end{array}$ & $\begin{array}{c}16(76.2 \%) \\
5(23.8 \%)\end{array}$ & $\begin{array}{c}41(87.2 \%) \\
6(12.8 \%)\end{array}$ & $\begin{array}{c}69(93.2 \%) \\
5(6.8 \%)\end{array}$ & $\begin{array}{c}2(6.7 \%) \\
28(93.3 \%)\end{array}$ \\
\hline $\begin{array}{l}\text { Tumour size } \\
<2 \mathrm{~cm} \\
\geq 2 \mathrm{~cm} \\
\text { unk }\end{array}$ & $\begin{array}{c}31(18.0 \%) \\
132(76.8 \%) \\
9(5.2 \%)\end{array}$ & $\begin{array}{c}4(19.0 \%) \\
17(81.0 \%) \\
0\end{array}$ & $\begin{array}{c}12(25.5 \%) \\
35(74.5 \%) \\
0\end{array}$ & $\begin{array}{c}14(18.9 \%) \\
53(71.6 \%) \\
7(9.5 \%)\end{array}$ & $\begin{array}{c}1(3.3 \%) \\
27(90.0 \%) \\
2(6.7 \%)\end{array}$ \\
\hline $\begin{array}{l}\text { Myoinvasion } \\
\text { None } \\
<50 \% \\
\geq 50 \%\end{array}$ & $\begin{array}{l}39(22.7 \%) \\
82(47.7 \%) \\
51(29.6 \%)\end{array}$ & $\begin{array}{c}5(23.8 \%) \\
11(52.4 \%) \\
5(23.8 \%)\end{array}$ & $\begin{array}{l}13(27.7 \%) \\
19(40.4 \%) \\
15(31.9 \%)\end{array}$ & $\begin{array}{l}16(21.6 \%) \\
37(50.0 \%) \\
21(28.4 \%)\end{array}$ & $\begin{array}{c}5(16.7 \%) \\
15(50 \%) \\
10(33.3 \%)\end{array}$ \\
\hline $\begin{array}{l}\text { FIGO stage } \\
\text { I-II } \\
\text { III-IV }\end{array}$ & $\begin{array}{l}140(81.4) \\
32(18.6 \%)\end{array}$ & $\begin{array}{c}17(81.0 \%) \\
4(19.0 \%)\end{array}$ & $\begin{array}{c}43(91.5 \%) \\
4(8.5 \%)\end{array}$ & $\begin{array}{l}64(86.5 \%) \\
10(13.5 \%)\end{array}$ & $\begin{array}{l}15(50.0 \%) \\
15(50.0 \%)\end{array}$ \\
\hline $\begin{array}{l}\text { LVI } \\
\text { Negative } \\
\text { Positive }\end{array}$ & $\begin{array}{c}117(68.0 \%) \\
55(32.0 \%)\end{array}$ & $\begin{array}{c}15(71.4 \%) \\
6(28.6 \%)\end{array}$ & $\begin{array}{l}31(66.0 \%) \\
16(34.0 \%)\end{array}$ & $\begin{array}{l}59(79.7 \%) \\
15(20.3 \%)\end{array}$ & $\begin{array}{l}12(40.0 \%) \\
18(60.0 \%)\end{array}$ \\
\hline $\begin{array}{l}\text { Pelvic and Para- } \\
\text { aortic LND } \\
\text { Yes } \\
\text { No }\end{array}$ & $\begin{array}{c}171(99.4 \%) \\
1(0.6 \%)\end{array}$ & $21(100 \%)$ & 47 (100\%) & $74(100 \%)$ & $\begin{array}{c}29(96.7 \%) \\
1(3.3 \%)\end{array}$ \\
\hline $\begin{array}{l}\text { Presence of LN } \\
\text { metastases }\end{array}$ & 31 (18.1\%) & 3 (14.2\%) & 7 (14.9\%) & $8(10.8 \%)$ & $13(44.8 \%)$ \\
\hline
\end{tabular}

LN, lymph node; LND, lymph node dissection; LVI, lymphovascular invasion; unk, unknown.

By multivariate analysis, molecular subtype and CA 125 $>25 \mathrm{kU} / \mathrm{L}$ were significantly associated with LN metastases ( $p=0.021$ and $p=0.022$ respectively) compared to histotype, which showed no significant association with LN status $(p=0.24)$.

Conclusion* EC molecular subtype significantly associates with LN metastases and offers objective, reproducible, and prognostic information from diagnostic specimens. Pre-operative knowledge of molecular subtype can guide biologicallyinformed approaches to LN sampling, particularly for patients with high molecular risk (p53abn) EC.

\section{THE ROLE OF PROGNOSTIC RISK FACTORS IN ENDOMETRIAL CANCER RECURRENCE: A RETROSPECTIVE STUDY}

${ }^{1} \mathrm{G}$ Spagnol*, ${ }^{2}$ Pezzani, ${ }^{2} \mathrm{G}$ Corrao, ${ }^{2} \mathrm{~S}$ Gava, ${ }^{2} \mathrm{~V}$ Bernardini, ${ }^{2} \mathrm{~S}$ Kilzie, ${ }^{3} \mathrm{G}$ Artioli, ${ }^{1} \mathrm{G}$ Bonaldo, ${ }^{1} \mathrm{M}$ Noventa, ${ }^{1} \mathrm{~S}$ Carlo, ${ }^{3} \mathrm{E}$ Busato. ${ }^{1} \mathrm{Cl}$ linic of Gynecology and Obstetrics, University of Padua, Padua, Italy, Department of Women and Children's Health; ${ }^{2}$ Treviso Regional Hospital, Treviso, Italy, Department of Obstetrics and Gynecology; ${ }^{3}$ Treviso Regional Hospital, Treviso, Italy, Department of Oncology

\subsection{6/ijgc-2021-ESGO.127}

Introduction/Background* Histopathologic type, grading, myometrial invasion, lymphovascular space invasion (LVSI) and 